AperTO - Archivio Istituzionale Open Access dell'Università di Torino

\title{
CFTR dysfunction in cystic fibrosis and chronic obstructive pulmonary disease
}

\section{This is the author's manuscript}

Original Citation:

Availability:

This version is available http://hdl.handle.net/2318/1686427

since 2019-05-15T14:05:34Z

Published version:

DOI:10.1080/17476348.2018.1475235

Terms of use:

Open Access

Anyone can freely access the full text of works made available as "Open Access". Works made available under a Creative Commons license can be used according to the terms and conditions of said license. Use of all other works requires consent of the right holder (author or publisher) if not exempted from copyright protection by the applicable law. 


\section{CFTR dysfunction in Cystic Fibrosis and Chronic Obstructive Pulmonary Disease}

Elena Fernandez Fernandez ${ }^{1 *}$, Chiara de Santi ${ }^{1 *}$, Virginia De Rose ${ }^{2}$, Catherine M. Greene ${ }^{1}$

*equal contribution.

\section{Affiliations:}

1. Lung Biology Group, Department of Clinical Microbiology, RCSI Education \& Research Centre, Beaumont Hospital, Dublin 9, Ireland. Phone +353-1-8093712

2. Department of Clinical and Biological Sciences, University of Torino, A.O.U. S. Luigi, Regione Gonzole 10, 10043 Orbassano, Torino, Italy. Phone +353-1-8093712. Phone +390119038674

Author email addresses: elenaffernandez@rcsi.ie; chiaradesanti@rcsi.ie; virginia.derose@unito.it; cmgreene@rcsi.ie

Corresponding author: cmgreene@rcsi.ie Phone +353-1-8093712 ORCiD 0000-0003-2549-2569 


\section{Abstract}

Introduction: Obstructive lung diseases such as cystic fibrosis (CF) and chronic obstructive pulmonary disease (COPD) are causes of high morbidity and mortality worldwide. CF is a multiorgan genetic disease caused by mutations in the cystic fibrosis transmembrane conductance regulator (CFTR) gene and is characterized by progressive chronic obstructive lung disease. Most cases of COPD are a result of noxious particles, mainly cigarette smoke but also other environmental pollutants.

Areas covered: Although the pathogenesis and pathophysiology of CF and COPD differ, they do share key phenotypic features and because of these similarities there is great interest in exploring common mechanisms and/or factors affected by CFTR mutations and environmental insults involved in COPD. Various molecular, cellular and clinical studies have confirmed that CFTR protein dysfunction is common in both the CF and COPD airways. This review provides an update of our understanding of the role of dysfunctional CFTR in both respiratory diseases.

Expert Opinion/Commentary: Drugs developed for people with CF to improve mutant CFTR function and enhance CFTR ion channel activity might also be beneficial in patients with COPD. A move toward personalized therapy using, for example, microRNA modulators in conjunction with CFTR potentiators or correctors, could enhance treatment of both diseases.

Key words: Cystic fibrosis transmembrane conductance regulator (CFTR), lung disease, cystic fibrosis (CF), chronic obstructive pulmonary disease (COPD), microRNA.

Funding details: This work was supported by the Cystic Fibrosis Foundation Therapeutics under Grant GREENE15XXO 


\section{Introduction}

Cystic fibrosis (CF) is a genetic disease caused by mutations in the cystic fibrosis transmembrane conductance regulator (CFTR) gene and remains one of the most common fatal hereditary disorders worldwide. Although CF is a complex multiorgan disease, morbidity and mortality are mainly determined by progressive chronic obstructive lung disease. The discovery of the CFTR gene set the stage for unravelling the molecular and cellular basis of CF lung disease. Chronic obstructive pulmonary disease (COPD) is a major and increasing global health problem and it is estimated to become the third leading cause of death worldwide by 2020 . The disease is caused by both genetic and environmental factors; in particular, cigarette smoking is the main risk factor for the development of COPD.

Although the pathogenesis and the pathophysiology of these diseases are different, they share key phenotypic features (Table 1), and recently great interest has arisen in investigating common mechanisms and/or factors that can be affected by CFTR mutations and environmental insults involved in CF and COPD, respectively. This article will focus on CFTR in CF and COPD, centering on similarities and differences in CFTR expression and function between both diseases.

\section{The Cystic Fibrosis Transmembrane Conductance Regulator}

\subsection{CFTR - discovery and structure}

The CFTR gene (CFTR), which was mapped by positional cloning in 1985, is located on the long arm of chromosome 7 (7q31.2) and spans approximately $190 \mathrm{~kb}$ of genomic DNA. It has 27 exons of various sizes producing a mature mRNA transcript of around $6.2 \mathrm{~kb}$. In 1991, the cDNA sequence of CFTR was determined along with the locations of intron/exon boundaries [1]. CFTR encodes a single polypeptide chain of 1480 amino acids with a predicted molecular weight of $168 \mathrm{KDa}$ [2-4]. 


\subsection{CFTR protein - structure, expression and function}

Based on the sequence of the CFTR protein, a structure was proposed that showed similarity to proteins in the ATP-binding cassette (ABC) transporter family [3]. The CFTR protein is embedded in the apical membrane of epithelial cells and is made up of distinct structural domains, including two membrane-spanning domains (MSDs), two nucleotide binding domains (NBD) and a regulatory domain (R) (Figure 1). The R region is unique to CFTR, as are the long $\mathrm{N}$ - and $\mathrm{C}$ - terminal extensions, which are 80 and 30 residues in length, respectively. The $R$ domain is phosphorylated by the protein kinases A (PKA), C (PKC) and 2 (CK2) and is dependent on the presence of intracellular ATP [5]. CFTR activity is likely regulated by a large number of other proteins including post-synaptic density 95/disc-large/zona occludens 1 (PDZ) interacting proteins and sulfate transporter and anti-sigma factor antagonist (STAS) domain interactors [6]. The NBDs contain conserved motifs for ATP binding and hydrolysis.

CFTR is expressed in the epithelia of many exocrine tissues including the airways, lung, pancreas, liver, intestines, vas deferens and sweat gland/duct [7]. The protein product primarily functions as an ion channel that, in concert with the $\mathrm{Ca}^{2+}$-activated $\mathrm{Cl}^{-}$channel $(\mathrm{CaCC})$, secretes $\mathrm{Cl}^{-}$; this process contributes to hydration of airway mucus. It also has the additional ability to transport bicarbonate [8] and glutathione [9]. CFTR also plays a role in regulating the activity of other membrane proteins including the epithelial $\mathrm{Na}^{+}$channel $(\mathrm{ENaC})$ and there is evidence which suggests that mutated forms of CFTR are unable to restrict salt absorption through ENaC [10]. Indeed, CFTR and ENaC play the most important roles in maintaining fluid homeostasis by controlling the movement of water through the epithelium, thus regulating the hydration of the epithelial surface in many organs. Prior to the effective treatment of the gastrointestinal manifestations of $\mathrm{CF}$, the Gl tract was responsible for most of the lethality in CF. Currently most focus is on CFTR in the airways where epithelial CFTR dysfunction leads to airway surface liquid (ASL) volume depletion due to an imbalance between CFTR-mediated $\mathrm{Cl}^{-}$secretion and ENaC-mediated $\mathrm{Na}^{+}$absorption [11]. 


\subsection{CFTR processing}

A large spectrum of regulatory and signaling events is involved in CFTR biogenesis and trafficking. Quality control mechanisms modulate CFTR folding, stability and targeting to the plasma membrane [12]. The CFTR polypeptide chain emerging from the ribosome is co-translationally inserted into the endoplasmic reticulum (ER) through the sequential and coordinated action of signal and stop transfer signals through the translocon. After insertion into the ER membrane, the nascent CFTR protein emerges into the ER lumen. Here, glycosylation plays a pivotal role in the quality control process that assesses the folding status of proteins destined for the secretory pathway (reviewed in [13]). Early studies using gel electrophoresis identified three forms of CFTR; a fully glycosylated band C (mature), a core-glycosylated band B (immature), and a non-glycosylated band A.

The regulation of CFTR trafficking, turnover and retention at the surface of epithelial cells is a complex process. Several members of the Ras superfamily of small GTPases, such as Rab and Rho subfamilies have been implicated in the regulation of these late stages of plasma membrane trafficking (reviewed in [14]). Ten percent of plasma membrane CFTR is constitutively internalized each minute by endocytosis through clathrin-coated vesicles [15]. Following internalization, CFTR is recycled back to the cell surface with high efficiency [16].

\section{Cystic fibrosis (CF)}

$\mathrm{CF}$ is the most common fatal autosomal recessive disease among Caucasians and is found in all ethnic groups [2,3]. It is estimated there are more than 70,000 CF patients worldwide (www.cff.org). Chronic obstructive lung disease is the major cause of morbidity and is responsible for $80 \%$ of the mortality in CF [18]. The lung disease is characterised by chronic lung infection and inflammation, leading to irreversible lung damage and death. CF is caused by mutations in CFTR (see below). Although its pulmonary manifestations are responsible for the major morbidity and mortality, the disease is also 
characterised by an array of extrapulmonary clinical manifestations including exocrine pancreatic insufficiency and CF-related diabetes, meconium ileus (intestinal obstruction) in the neonatal period, liver involvement and, in a small proportion of patients, cirrhosis and portal hypertension. The principal clinical manifestations of $\mathrm{CF}$ are an elevated sweat chloride concentration, progressive pulmonary disease, exocrine pancreatic insufficiency and male infertility (recently reviewed in [19]). The primary defect in the CFTR gene leads to the major pulmonary pathology of CF, inadequate homeostasis of the ASL and consequently, impaired mucociliary clearance. This leads to an increased mucus viscosity, chronic bacterial infection and inflammation, bronchiectasis and ultimately respiratory failure.

CF was first described as a disease entity in 1938 by Dorothy Andersen, who published a detailed autopsy study of malnourished infants and characterised a disease of mucus plugging of glandular ducts distinct from others with coeliac syndrome [20]. At that time, most people with CF died shortly after diagnosis. However, significant improvements have been made in recent decades regarding life expectancy, especially since the introduction in the mid-1980s of acid-resistant pancreatic enzyme replacement therapies to compensate for pancreatic insufficiency [21]. According to the US CF Foundation Patient Registry, the median predicted survival age for patients increased from 25 years in 1985 to over 41 by 2012 [22]. Since then there have been great advances in CF disease management with the introduction of CFTR potentiatior and corrector drugs in the recent past [23-25]

\subsection{Clinical effects of dysfunction CFTR in CF}

During the last decade, huge progress has been made in understanding the cellular and molecular basis of CF lung disease and their effects on the pathophysiology of the disease. As already pointed out, this led to earlier diagnosis, better patient management and to a remarkable improvement 
in survival and quality of life for CF patients. Furthermore, innovative and specific therapies targeting the CFTR defect have been developed, in the context of personalized medicine [23-25].

Dysfunction of the CFTR protein in the airway of CF patients affects a multitude of cellular functions, including transmembrane transport of $\mathrm{Cl}^{-}, \mathrm{Na}^{+}$, and bicarbonate, mucin secretion and inflammatory signaling. This predisposes CF patients to chronic airway infection and associated airway inflammation, leading to progressive lung injury and airflow obstruction [26].

Defective $\mathrm{Cl}^{-}$and bicarbonate transport into the airway lumen, consequent to dysfunction of the CFTR protein, results in dehydration and acidification of ASL, production of viscous, acidic, and altered secretions, and impairment in mucociliary clearance $[27,28]$. There is emerging evidence of the key role of bicarbonate secretion in regulating local $\mathrm{pH}$ and the airway milieu: bicarbonate drives ionic content and fluids on epithelial surfaces, allows mucins to unfold and contributes to innate immunity. When CFTR is dysfunctional, lack of bicarbonate and chloride secretion disrupts these normal processes and creates an abnormal airway milieu, with impaired innate host defenses, that favours chronic airway infection and inflammation leading to progressive lung injury and ultimately to respiratory failure. A reduction in ASL pH in the CF pig model has recently been shown to decrease antimicrobial activity which was restored by directly increasing $\mathrm{pH}$ [29]. In addition to the alterations of the airway milieu, there is evidence that CFTR dysfunction itself is associated with defects in innate and adaptive immune defenses that lead to impaired bacterial clearance and dysregulated inflammation [30, 31]. Chronic airway inflammation in CF is mainly characterized by a persistent and marked neutrophil recruitment into the airways. These cells release a variety of oxidants and granule-associated enzymes that contribute to structural lung damage and lung function decline as well as to the maintenance of chronic pulmonary infection. Neutrophilic airway inflammation is further augmented after the onset of chronic bacterial infection and during the repeated episodes of acute infection that characterize the natural history of the disease. In this context, the inflammatory response in the CF lung is non-resolving and 
self-perpetuating, and a vicious cycle of inflammation and infection occurs that further contributes to irreversible lung damage and disease progression $[32,33]$. Additional ion channels to CFTR, are present on the apical surface of airway epithelium and are involved in maintaining ASL volume and composition. These include ENaC, CaCCs and the solute carrier 26A (SLC26A) family of anion exchangers; novel treatments aimed at modulating these alternative channels and transporters could provide an attractive alternative strategy to correct the ASL abnormalities evident in CF [22, 24].

\section{Mechanisms of CFTR dysfunction in CF}

\subsection{Mutation classes}

To date, almost 2000 different mutations of the CFTR gene have been identified and collected by the international CF genetics research community [34]. The most common mutation ( $85 \%$ of patients worldwide) is a three base deletion that removes a phenylalanine at position 508 (p.Phe508del) of the CFTR protein. CFTR mutations can be divided into six major classes according to their deleterious effect on the protein. Classes I-III are associated with no effective CFTR function, while classes IV-VI have residual function. However, very recently, it has been proposed that CFTR mutations can also provide the scientific basis for mutation-specific corrective therapies and thus, are grouped into seven classes according to their functional defect [24]. The underlying aim of this stratification of CFTR mutations is to apply the same therapeutic correction to the basic defect in each class. These mutations are classified as follows:

* Class I mutations result in the total or partial lack of production of a functional CFTR protein. Such mutations may arise either due to premature termination signals because of splice site abnormalities, frameshifts due to insertions or deletions, or nonsense mutations, for example G542X or R1162X.

* Class II mutations are associated with defective processing due to misfolding of the protein and may be found within any domain of the CFTR protein. The misfolded protein is retained in the endoplasmic 
reticulum and re-translocated to the cytoplasm, where it is degraded by the ubiquitin/proteasome pathway. This class includes the most common mutation, p.Phe508del. It has been shown that p.Phep.Phe508del leads to energetic and kinetic instability of the NBD1 domain due to improper local folding [35].

* Class III mutations are frequently located in the ATP binding domains (NBD1 and NBD2) and are referred to as gating mutations. They are missense mutations which produce a protein that is efficiently inserted in the apical membrane at normal levels but is resistant to activation by PKA. The best known example is G551D [36].

* Class IV mutations are mostly located within membrane spanning domains implicated in the constitution of the channel pore. The missense mutations located in these regions produce a protein that is inserted into the membrane appropriately and retains CAMP-dependent chloride channel activity, but has reduced channel conductance [37].

* Class V mutations reduce the total amount of CFTR protein by affecting pre-mRNA splicing. These mutations allow synthesis of some normal CFTR mRNA (and protein), although at very low levels [38]. This class also includes promoter mutations that reduce transcription and mutations that encode amino acids that cause inefficient protein maturation [39].

* Class VI mutants have impaired CFTR protein plasma membrane stability [40].

* A newly described class, Class VII, includes mutants 'unreachable by pharmacological means per se'. [24].

Despite efforts by the scientific community to classify all CFTR mutations, this system and others are limited in their scope. For instance, one single mutation can show different defects and can therefore be classified into more than one class (e.g. p.Phe508del (Class II, III and VI)). It must also be considered that at present the majority of CFTR mutations have not been functionally characterized. 
Functional studies on agnostic class VII mutations will facilitate a clearer understanding of these specific defects.

\subsection{Other mechanisms affecting CFTR expression in CF}

Other mechanisms that can affect CFTR expression in CF include transcriptional and posttranscriptional events. Expression of CFTR is normally tightly controlled and is spatially and temporally regulated, although CFTR transcription can begin at different start sites depending on the tissue or developmental stage in question [41]. CFTR can also be post-transcriptionally regulated by microRNAs (miRNAs) [41-43].

Expression of miRNA is known to be altered in many pathological states, including lung inflammation and disease. Our group was the first to examine miRNA expression in CF [44]. Numerous miRNAs had altered expression between CF and non-CF bronchial epithelium, and some of the altered miRNAs were predicted to regulate CFTR. Various other published studies have examined the role of miRNAs in the direct or indirect control of wild type (wt) or mutant CFTR expression [41-43, 45-50]. Based on these published studies the lead miRNAs that have been experimentally validated as regulators of wt and/or p.Phe508del CFTR are miR-101, miR-145, miR-223, miR-494 and miR-509-3p. It may be feasible to modulate CFTR expression in the future using miRNA-targetting medicines [51].

\section{Chronic obstructive pulmonary disease (COPD)}

\subsection{COPD}

COPD is characterized by a persistent airflow limitation that is usually progressive and associated with an enhanced chronic inflammatory response of the airways and lungs to noxious particles or gases (e.g. tobacco smoke)[52]. COPD is the fifth leading cause of death in high-income countries, however globally it is the third leading cause of death [53], resulting in 2.9 million deaths 
annually and accounting for $6 \%$ of the global healthcare budget $[54,55]$. Worldwide, more than 12 million people are currently diagnosed with COPD and an additional 12 million are likely to have the disease but are undiagnosed. The disease kills more than 120,000 Americans each year and the number of people with COPD is increasing [56]. Each year, about 300,000 people die in Europe due to COPD [77], and the morbidity, disability, economic and social impact of COPD is substantial and increasing. The main cause of COPD in Europe and the USA is cigarette smoking or exposure to second-hand smoke/passive smoke exposure. According to WHO (World Health Organization) estimates in 2005, worldwide 5.4 million people died due to tobacco use that year and tobacco-related deaths are projected to increase to 8.3 million deaths per year by 2030 [58].

In low-income countries exposure to smoke from biomass fuels used for cooking and heating is a major cause of COPD. Almost 3 billion people worldwide use biomass and coal as their main source of energy for household needs. In these communities, indoor air pollution is responsible for a greater fraction of COPD risk than smoking or outdoor air pollution and accounts for the high prevalence of COPD among non-smoking women in parts of the Middle East, Africa and Asia. Indoor air pollution resulting from the burning of wood and other biomass fuels is estimated to kill two million women and children each year. Other risk factors for COPD include occupational dusts and chemicals (such as vapours, irritants, and fumes) and frequent lower respiratory infections during childhood.

\subsection{Clinical relevance of CFTR dysfunction in COPD}

Although the pathogenesis and the pathophysiology of CF and COPD are different, they share key phenotypic features and both diseases are associated with neutrophilic inflammation in the airway lumen, progressive airflow obstruction and recurrent exacerbations that significantly contribute to morbidity and mortality. These similarities raise the possibility that common mechanism may contribute 
to both CF and COPD. Growing evidence suggests that cigarette smoking induces an acquired CFTR dysfunction in patients with a normal CFTR genotype [59-61].

The first evidence that airway ion secretion may be altered in cigarette smokers was reported by Welsh [62] well before CFTR had been discovered. Several studies have subsequently confirmed that sustained exposure to cigarette smoke, or cigarette smoke extract (CSE) reduces CFTR expression and function in vivo, in the airways of smokers and patients with COPD, and in airway epithelial cells in vitro [62-66]. Furthermore, it has been shown that CSE is capable of inducing a delayed mucociliary transport in vitro, and a marked increase in mucus expression both in vitro and in vivo [67-69]. Interestingly, in patients with COPD, significant correlations have been shown between clinical manifestations such as dyspnea and symptoms of chronic bronchitis and the levels of CFTR suppression in their lower airways [64]; furthermore, the density of CFTR protein expression in the lung of patients with emphysema was shown to be significantly correlated with lung function and inversely correlated with COPD stage [70]. In mice overexpressing the $\beta$ subunit of $\mathrm{ENaC}(\beta E N a C)$ a decrease of CFTR function was reported to aggravate the airway mucus obstruction and mortality, consistent with a role of CFTR in defining the severity of COPD manifestations [71]. These studies strongly suggest that CFTR dysfunction induced by cigarette smoke likely plays a role in COPD pathogenesis and may represent a potential target for the development of novel therapeutic approaches. Several independent mechanisms have been proposed to account for the effects of cigarette smoking on CFTR, including malfunctioning transcription, alteration of protein stability and function, and accelerated internalization of CFTR channels from the apical plasma membrane $[63,64,66,67,69,70]$. The acquired CFTR dysfunction results in a reduced capacity of the airway epithelium to secrete fluid and leads to ASL dehydration, and together with mucin hypersecretion, is predicted to contribute to the development of chronic bronchitis in smokers and COPD patients, and to aggravate mucus hyperconcentration and plugging in COPD airways $[69,72,73]$. 
Chronic mucus hypersecretion is present in most COPD patients and is independently associated with lung function decline; therefore, the possibility of treating mucus hypersecretion by therapeutic strategies that correct CFTR dysfunction is clinically relevant.

Bicarbonate secretion is also mediated by CFTR [8] and plays a crucial role in regulating local pH and the airway milieu; it also allows mucins to unfold and contributes to innate immunity [26-28]; tobacco smoke exposure induces a decrease of bicarbonate secretion presumably resulting in acidification of the ASL $[74,75]$. The loss of CFTR function induced by cigarette smoke exposure is also associated with loss of lipid raft CFTR, increased membrane ceramide accumulation and increased apoptosis [76]. It has also been shown that altered CFTR lipid rafts in macrophage exposed to cigarette smoke impair macrophage bacterial phagocytosis and killing, which might contribute to recurrent bacterial infections in the disease [77]. Interestingly, it has been reported that COPD patients with a history of disease exacerbations during the past year exhibited less CFTR function than those without a history of exacerbations, further emphasizing the clinical consequences associated with acquired CFTR dysfunction in COPD [67].

Several toxic agents in cigarette smoke are capable of inducing changes in CFTR gene expression, protein stability and anion conductance. Current evidence implicates acrolein and cadmium as cigarette smoke constituents that inhibit CFTR function in vitro [78-80]. Cadmium was also shown to accumulate in the lungs of COPD patients with GOLD stage IV (The Global Initiative for Chronic Obstructive Lung Disease) and negatively correlate with CFTR expression, suggesting that this heavy metal may act as a toxin that continuously inhibits CFTR expression in severe COPD patients [81]. Interestingly, CFTR dysfunction has also been shown to be induced by oxidative stress, neutrophilic inflammation, and hypoxia, conditions that are known to be associated with advanced COPD. Increased levels of neutrophil elastase that have been reported in COPD airways may also play a role in acquired CFTR dysfunction observed in COPD patients, as neutrophil elastase can induce CFTR degradation [82]. 
CFTR dysfunction has also been documented at a systemic level in smokers with or without COPD: Raju and colleagues reported that healthy smokers and smokers with COPD had elevated sweat chloride levels compared with normal control subjects, indicating that sweat glands are also affected by cigarette smoking and COPD [78]. In the same study, by using intestinal current measurements, a $65 \%$ decrease in CFTR function in the rectal epithelium was observed in smokers compared with never smokers, confirming an acquired extrapulmonary CFTR dysfunction in smokers.

Thus, there is now a large body of evidence indicating that acquired CFTR dysfunction induced by cigarette smoke may contribute to COPD pathogenesis, particularly in patients with the chronic bronchitis phenotype. Taken together, recent studies in animal models, human airway epithelia, and patients support the concept that ASL dehydration in combination with mucus hypersecretion and a reduced capacity to hydrate secreted mucins, is an important disease mechanism leading to mucociliary dysfunction and mucus plugging, which in turn trigger inflammation and facilitate bacterial infection.

\section{Mechanisms of CFTR dysfunction in COPD}

COPD and cystic fibrosis share many clinical manifestations, including airway obstruction and inflammation, chronic bronchial infections and acute respiratory exacerbations. This could be due to the presence of overlapping pathways among these two respiratory diseases, and an ongoing body of evidences suggests that a key player could be dysfunctional CFTR. Several clinical studies have reported that CFTR activity is impaired in the lower airways of smokers with or without COPD [63, 64]. Moreover, these studies showed that CFTR dysfunction due to cigarette smoke (CS) is associated with such clinical characteristics as chronic bronchitis and dyspnea [64].

While CS-induced CFTR dysfunction in clinical studies is often measured in terms of nasal potential difference, it is primarily measured in vitro as a reduced short-circuit current $\left(I_{s c}\right)$ when cells are mounted in Ussing chambers and treated with CS, as shown by very early reports dated even before 
the discovery of the CFTR gene [62]. The negative effect of CS exposure on CFTR-dependent $I_{s c}$ has repeatedly been reported by others $[65,67,78,83,84]$. The consequences of an impaired chloride ion conductance following CS exposure resembled the pathological features of CF cells, i.e. decreased ciliary function, dehydration and reduction of ASL, increased mucin production and impaired mucus clearance. Indeed, following CS exposure many authors observed a decrease in ASL height $[66,70]$ and in mucociliary transport in vitro [67], while mucin secretion was higher when bronchial epithelial cells were treated with CS compared to controls [65].

The molecular and cellular mechanisms that lead to CFTR dysfunction in smokers are currently under investigation and they could represent, when specifically addressed, a target for the development of novel therapeutic strategies in COPD and other respiratory diseases where smoke plays a central role. For this reason, several research groups have aimed to investigate the mechanisms that could be responsible for CS-induced CFTR dysfunction in vitro and reported them at different stages of the gene regulatory network.

\subsection{Transcriptional and post-transcriptional effects}

While investigating the role of CFTR in oxidative stress in the context of glutathione transport [85], Cantin and collaborators discovered that the rate of CFTR mRNA degradation was accelerated in CFTR-expressing cells (T84 and Calu-3) treated with oxidants compared to controls [63]. When the same cells were exposed to CS, a well-known source of oxidants with more than $10^{15}$ radicals/puff [86], CFTR mRNA levels were decreased compared to control-treated cells as measured by Northern blotting [63]. Interestingly, conserved antioxidant response elements (AREs) were identified within the CFTR promoter and were reported to recruit $\mathrm{Nrf2}$, a transcription factor that plays a key protective role against oxidative stress [87]. Zhang and co-authors showed that bronchial epithelial cells treated with the Nrf2 agonist sulforaphane, a naturally occurring antioxidant, respond with an initial increase of CFTR mRNA 
expression due to recruitment of $\mathrm{Nrf2}$ in the nucleus, followed by a time-dependent decrease [88]. These data collectively show that CFTR mRNA expression can be modulated subsequent to oxidative stress that notoriously persists in cigarette smokers.

At a post-transcriptional level, CFTR mRNA is known to be negatively regulated by a set of lead miRNAs including miR-101, miR-144 and miR-223 [89]. Interestingly, CS-exposed bronchial epithelial cells express higher levels of miR-101 and miR-144 than control-treated cells, and the results for miR101 are paralleled in vivo in mice treated with CS and in lung tissues of COPD patients [51]. Interestingly, miR-223 is also up-regulated in smokers with COPD [90] overall, suggesting that alteration in miRNA expression could contribute to CS-induced CFTR dysfunction.

\subsection{Post-translational effects}

Independent of mRNA regulation, CS has been shown to negatively affect CFTR protein levels directly. Clunes and collaborators observed by immunofluorescence that CFTR was completely absent from the apical membrane of primary bronchial epithelial cells after only 10 minutes of CS exposure [66]. The authors reported that following CS treatment CFTR was removed from the plasma membrane, internalised and trafficked to an aggresome-like perinuclear compartment, possibly subsequent to quality control mechanisms. Further studies revealed that CS treatment increased cytosolic $\mathrm{Ca}^{2+}$ levels and CS-induced CFTR internalisation could be reversed when cells were pre-incubated with the $\mathrm{Ca}^{2+}$ chelator BAPTA-AM [70]. While neither endoplasmic reticulum nor mitochondria were found to be the sources of the increased $\mathrm{Ca}^{2+}$ levels, further experiments where lysosomal transport was inhibited by bafilomycin showed a rescue of the CS-induced CFTR internalization, suggesting that this phenomenon is accompanied by $\mathrm{Ca}^{2+}$ release from the lysosomes.

Some research groups also focused on particular components of CS and found interesting mechanisms that affect CFTR function. As mentioned, cadmium, a toxic heavy metal abundantly present 
in CS, can decrease CFTR protein levels in human airway epithelium in vitro and in mice in vivo [79]. The mechanism involves cadmium-induced ubiquitination of CFTR protein, leading to its degradation; this mechanism can be prevented by the antioxidant $\alpha$-tocopherol. The toxic aldehyde acrolein was reported to decrease CFTR function in primary murine nasal septal epithelia [83] and human bronchial epithelial cells [78]. Further studies showed that acrolein biochemically altered CFTR protein and that these covalent modifications could change the structure of the channel, thereby impairing its function and possibly its stability on the cell surface [61].

Interestingly, second-hand smoke has also been reported to affect CFTR function in vitro [91]. Using a custom-designed exposure system where the concentration of carbon monoxide (CO) was kept around $5 \mathrm{ppm}$, (i.e. the levels of CO estimated as second-hand smoke in households and cars), bronchial epithelial cells were exposed to CS. Cells showed a reduced forskolin-stimulated $I_{s c}$ paralleled by a decrease in CFTRinh172-sensitive $I_{s c}$, suggesting that the chloride transport through CFTR was impaired.

In this context, it is reasonable to hypothesise that drugs that attempt to restore CFTR chloride channel function could be attractive for COPD individuals with acquired CS-induced CFTR dysfunction. Sloane and collaborators showed that the clinically approved CFTR potentiator ivacaftor (VX-770) was able to rescue CS-mediated inhibition of CFTR in non-CF bronchial epithelial cells in terms of CFTRdependent $\mathrm{I}_{\mathrm{sc}}$, ASL height and mucociliary transport [67]. The same results were confirmed in another recent study [78] and demonstrate that CFTR potentiation could represent a therapeutic strategy for COPD. On the other hand, the bioactivity of drugs clinically approved for COPD patients could be explained by their role in recovering CFTR function after CS exposure. For example, the phosphodiesterase-4 inhibitor roflumilast was shown to activate the CFTR channel, as shown by the increase of $I_{s c}$ in bronchial epithelial cells treated with increasing concentrations of roflumilast [92]. This mechanism was due to an increase of intracellular CAMP levels following roflumilast treatment leading to increased phosphorylation (and thus activity) of the CFTR channel and eventually to a recovery of the 
CS-induced CFTR inhibition. These data were confirmed by others where roflumilast was also shown to rescue ASL dehydration [93] and partially restore ciliary dysfunction [94] caused by CS exposure in bronchial epithelial cells.

\section{Expert Commentary}

CFTR gene defects are responsible for CF and its pulmonary manifestations. It is becoming increasingly clear that CFTR dysfunction in smokers may contribute to the pathophysiology of COPD, however direct clinical evidence for this has yet to be published. Nonetheless it is highly likely that drugs developed for people with CF to improve mutant CFTR function and/or enhance CFTR ion channel activity might also be beneficial in patients with COPD. Although a number of studies have shown the potential benefit of enhancing CFTR activity in vitro $[61,67,92-94]$ the challenge for researchers is to perform even more studies using a range of CFTR-targeted therapies and as wide range of CFTR functional outputs as possible. Collectively these would demonstrate whether enhancement of CFTR expression and function in COPD epithelium has true efficacy. This data would encourage clinical studies of CFTR-targeted therapies in COPD.

Within the CF field combinations of CFTR-directed therapies can be tested for CFTR correction in ex vivo models using a patient's own cells e.g. intestinal organoids, nasospheroids. For example, patientderived ex vivo rectal biopsies can be tested for CFTR function and the effects of CFTR modulators can be assessed on these epithelia. A newer method employs the use of three-dimensional epithelial organoids derived from primary culture of rectal biopsies [95-103]. Forskolin-induced swelling of these organoids can assess CFTR function in response to treatment with approved or investigational drugs. A similar strategy could be implemented for COPD sufferers. However, whether intestinal organoids directly recapitulate the functional characteristics of airway epithelium, whether CFTR activity in these organoids is an accurate predictor of lung disease, and if intestinal epithelium can actually predict 
airway response to CFTR drugs is not known. Thus nasal-derived cells cultured into nasospheroids may be a more appropriate model to use [104]. This personalised medicine approach would correctly assess whether an individual patient will likely respond to a given CFTR modulator or respond better versus another choice. Ultimately, a move toward personalised therapy using, for example miRNA modulators in conjunction with CFTR potentiators or correctors, could enhance treatment of both diseases.

The rapidly advancing field of induced pluripotent stem cell (iPSC) technology can also help to solve the challenge of CFTR dysfunction in COPD. The generation of human iPSCs from blood has become a manageable feat [105-107]. These cells can be differentiated into pulmonary cells and be used to assess CFTR function as well as for the validation of candidate drugs using electrophysiological assays that can quantify CFTR function [108-110]. However, it is important to note that the ex vivo state of COPD iPSCs may not represent CFTR dysfunction in COPD patients because the culturing environment no longer contains the harmful noxa. Nonetheless, iPSCs will be a useful tool for drug discovery.

Whilst existing CFTR modulator drugs, in theory, can now be used to treat almost $90 \%$ of people with $\mathrm{CF}$, researchers continue to develop novel alternative therapeutics that could be used independently to treat CF or as adjunct therapies to be co-administered to patients with CF under certain specific conditions. Adjunct therapies need to be considered for various reasons including, for example, to address impaired innate immune mechanisms and intrinsic defects in CF neutrophils [30] or microRNAs which are affected by infection or inflammation that can impact on CFTR drug efficacy. Thus, the efficacy of CFTR modulators could be enhanced by co-administration with, for example, nebulised nanoparticles encapsulating microRNA-modulating drugs that enhance CFTR expression.

Although CF is a rare disease, it is tantalizing to imagine that drugs developed specifically to enhance the health and quality of life of people with CF, could have therapeutic benefit for the vast number of people suffering from COPD worldwide. 


\section{Five-year view}

The next five years is likely to see a flurry of developments in this area. Key amongst these will be clinical studies designed to clearly demonstrate that CFTR dysfunction contributes to the pulmonary manifestations of COPD and that it represents a drug target in COPD. Identifying which stage of disease and what class of patient would benefit from CFTR-targeted therapies will also need to be determined during or soon after this time. In line with this, we will no doubt see a series of publications reporting the effects of a selection of CFTR-targeted therapies on CFTR function in advanced ex vivo 3-D models of COPD epithelium and air-liquid interface epithelial cultures derived from patient iPSCs. Within this time period we may also begin to see clinical trials testing combinations of existing and new CFTR modulators in people with COPD. 


\section{Key Issues}

- Mutations in CFTR underpin the clinical manifestations of CF. The primary defect in the CFTR gene leads to the major pulmonary pathology of CF; inadequate homeostasis of the airway surface liquid and impaired mucociliary clearance. This leads to an increased mucus viscosity, chronic bacterial infection and inflammation, bronchiectasis and ultimately respiratory failure.

- CFTR mutations can be divided into six major classes according to their deleterious effect on the protein. Classes I-III are associated with no effective CFTR function, while classes IV-VI have residual function. A newly described class, Class VII, includes mutants 'unreachable by pharmacological means'.

- The main cause of COPD in Europe and the USA is cigarette smoking or second-hand smoke/passive smoke exposure. In low income countries exposure to smoke from biomass fuels used for cooking and heating is a major cause of COPD.

- Although the pathogenesis and pathophysiology of CF and COPD differ, they share key phenotypic features and because of these similarities there is great interest in exploring common mechanisms and/or factors affected by CFTR mutations and environmental insults involved in COPD.

- Various molecular, cellular and clinical studies have confirmed that CFTR protein dysfunction is common in both the CF and COPD airways.

- CFTR modulator therapies aim to enhance CFTR expression and function. These include potentiators, correctors, stabilisers and amplifiers and were developed for people with CF to improve mutant CFTR expression or function and/or enhance CFTR ion channel activity however they might also be beneficial in patients with COPD. 


\section{REFERENCES}

[1] Zielenski J, Bozon D, Kerem B, et al. Identification of mutations in exons 1 through 8 of the cystic fibrosis transmembrane conductance regulator (CFTR) gene. Genomics 1991;10:229-235.

[2] **Rommens JM, lannuzzi MC, Kerem B, et al. Identification of the cystic fibrosis gene: chromosome walking and jumping. Science 1989;245:1059-1065.

${ }^{* *}$ Seminal paper reporting the mapping of the CFTR gene.

[3] **Riordan JR, Rommens JM, Kerem B, et al. Identification of the cystic fibrosis gene: cloning and characterization of complementary DNA. Science 1989;245:1066-1073

**First report of cloning and characterisation of the human CFTR and F508del mutation.

[4] **Kerem B, Rommens JM, Buchanan JA, et al. Identification of the cystic fibrosis gene: genetic analysis. Science 1989;245:1073-1080.

**Third in series of 3 papers joinlty published in a sibgle issue of Science in 1989

[5] Cheng SH, Rich DP, Marshall J, et al. Phosphorylation of the R domain by cAMP-dependent protein kinase regulates the CFTR chloride channel. Cell 1991;66:1027-1036.

[6] Ko SBH, Zeng W, Dorwart MR, et al. Gating of CFTR by the STAS domain of SLC26 transporters. Nat Cell Biol 2004;6:343-350.

[7] Welsh MJ, Smith AE. Molecular mechanisms of CFTR chloride channel dysfunction in cystic fibrosis. Cell 1993;73:1251-1254.

[8] Poulsen $\mathrm{JH}$, Fischer $\mathrm{H}$, Illek $\mathrm{B}$, et al. Bicarbonate conductance and $\mathrm{pH}$ regulatory capability of cystic fibrosis transmembrane conductance regulator. Proc Natl Acad Sci U. S. A. 1994;91:53405344.

[9] Kogan I, Ramjeesingh M, Li C, et al. CFTR directly mediates nucleotide-regulated glutathione flux. EMBO J 2003;22:1981-1989.

[10] Stutts MJ, Canessa CM, Olsen JC, et al. CFTR as a CAMP-dependent regulator of sodium channels. 
Science 1995;269:847-850.

[11] Mall M, Grubb BR, Harkema JR, et al. Increased airway epithelial Na+ absorption produces cystic fibrosis-like lung disease in mice. Nat Med 2004;10:487-493.

[12] Wang X, Matteson J, An Y, et al. COPII-dependent export of cystic fibrosis transmembrane conductance regulator from the ER uses di-acidic exit code. J Cell Biol 2004;167:65-74.

[13] Skach WR. Defects in processing and trafficking of the cystic fibrosis transmembrane conductance regulator. Kidney Int 2000;57:825-831.

[14] Farinha CM, Matos P, Amaral MD. Control of cystic fibrosis transmembrane conductance regulator membrane trafficking: not just from the endoplasmic reticulum to the Golgi. FEBS J 2013;280:4396-4406.

[15] Prince LS, Peter K, Hatton SR, et al. Efficient endocytosis of the cystic fibrosis transmembrane conductance regulator requires a tyrosine-based signal. J Biol Chem 1999;274:3602-3609.

[16] Sharma M, Pampinella F, Nemes C, et al. Misfolding diverts CFTR from recycling to degradation: quality control at early endosomes. J Cell Biol 2004;164:923-933.

[17] Zhang Z, Chen J. Atomic Structure of the Cystic Fibrosis Transmembrane Conductance Regulator. Cell 2016;167:1586-1597.e9.

[18] Cantin AM, Hartl D, Konstan MW, et al. Inflammation in cystic fibrosis lung disease: pathogenesis and therapy. J Cyst Fibros 2015;14:1-12.

[19] Elborn JS. Cystic fibrosis. Lancet 2016;388:2519-2531.

[20] Andersen DH. Cystic Fibrosis of the pancreas and its relation to celiac disease. Am J Dis Child 1938;56:344.

[21] Mehta G, Macek M, Mehta A. Cystic fibrosis across Europe: EuroCareCF analysis of demographic data from 35 countries. J Cyst Fibros 2010;9:S5-S21.

[22] Cystic Fibrosis Foundation. Cystic Fibrosis Foundation Patient Registry 2012 Annual Data Report. 
$2012 ; 28$.

[23] Quon BS, Rowe SM. New and emerging targeted therapies for cystic fibrosis. BMJ 2016;352.

[24] De Boeck K, Amaral MD. Progress in therapies for cystic fibrosis. Lancet Respir Med. 2016;4:662674.

[25] McNally P, Greene CM. Cystic fibrosis: a model for precision medicine. Expert Review of Precision Medicine and Drug Development 2018;2:107-117 .

[26] Mall MA, Hartl D. CFTR: cystic fibrosis and beyond. Eur Respir J 2014;44:1042-1054.

[27] Birket SE, Chu KK, Liu L, et al. A Functional Anatomic Defect of the Cystic Fibrosis Airway. Am J Respir Crit Care Med 2014;190:421-432.

[28] Tang XX, Ostedgaard LS, Hoegger MJ, et al. Acidic $\mathrm{pH}$ increases airway surface liquid viscosity in cystic fibrosis. J Clin Invest 2016;126:879-891.

[29] Pezzulo AA, Tang XX, Hoegger MJ, et al. Reduced airway surface $\mathrm{pH}$ impairs bacterial killing in the porcine cystic fibrosis lung. Nature 2012;487:109-113.

[30] Hartl D, Gaggar A, Bruscia E, et al. Innate immunity in cystic fibrosis lung disease. J Cyst Fibros 2012;11:363-382.

[31] Cantin AM, Hartl D, Konstan MW, et al. Inflammation in cystic fibrosis lung disease: pathogenesis and therapy. J Cyst Fibros 2015;14:419-430.

[32] De Rose V. Mechanisms and markers of airway inflammation in cystic fibrosis. Eur Respir J 2002;19:333-340.

[33] Cohen-Cymberknoh M, Kerem E, Ferkol T, et al. Airway inflammation in cystic fibrosis: molecular mechanisms and clinical implications. Thorax 2013;68:1157-1162.

[34] ${ }^{*}$ Cystic Fibrosis Mutation Database: Statistics [Internet]. [cited 2017 Aug 9]. Available from http://www.genet.sickkids.on.ca/cftr/StatisticsPage.html

*Useful resource on frequencies of CFTR mutations 
[35] Protasevich I, Yang Z, Wang C, et al. Thermal unfolding studies show the disease causing F508del mutation in CFTR thermodynamically destabilizes nucleotide-binding domain 1. Protein Sci 2010;19:1917-1931.

[36] Delaney SJ, Alton EW, Smith SN, et al. Cystic fibrosis mice carrying the missense mutation G551D replicate human genotype-phenotype correlations. EMBO J 1996;15:955-963.

[37] Sheppard DN, Rich DP, Ostedgaard LS, et al. Mutations in CFTR associated with mild-disease-form Cl- channels with altered pore properties. Nature 1993;362:160-164.

[38] Ramalho AS, Beck S, Meyer M, et al. Five percent of normal cystic fibrosis transmembrane conductance regulator mRNA ameliorates the severity of pulmonary disease in cystic fibrosis. Am Respir Cell Mol Biol 2002;27:619-627.

[39] Taulan M, Lopez E, Guittard C, et al. First functional polymorphism in CFTR promoter that results in decreased transcriptional activity and Sp1/USF binding. Biochem Res Commun 2007;361:775781.

[40] Haardt M, Benharouga M, Lechardeur D, et al. C-terminal truncations destabilize the cystic fibrosis transmembrane conductance regulator without impairing its biogenesis. A novel class of mutation. J Biol Chem 1999;274:21873-21877.

[41] Viart V, Bergougnoux A, Bonini J, et al. Transcription factors and miRNAs that regulate fetal to adult CFTR expression change are new targets for cystic fibrosis. Eur Respir J 2015;45:116-128.

[42] Oglesby IK, Chotirmall SH, McElvaney NG, et al. Regulation of Cystic Fibrosis Transmembrane Conductance Regulator by MicroRNA-145, -223, and -494 Is Altered in $\Delta$ F508 Cystic Fibrosis Airway Epithelium. J Immunol 2013;190;3354-3362.

[43] Ramachandran S, Karp PH, Osterhaus SR, et al. Post-transcriptional regulation of cystic fibrosis transmembrane conductance regulator expression and function by microRNAs. Am J Respir Cell Mol Biol 2013;49:544-551. 
[44] Oglesby IK, Bray IM, Chotirmall SH, et al. miR-126 is downregulated in cystic fibrosis airway epithelial cells and regulates TOM1 expression. J. Immunol. 2010;184:1702-1709.

[45] *Gillen AE, Gosalia N, Leir S-H, et al. MicroRNA regulation of expression of the cystic fibrosis transmembrane conductance regulator gene. Biochem J 2011;438:25-32.

* First published report of microRNA regualtion of CFTR

[46] Megiorni F, Cialfi S, Dominici C, et al. Synergistic Post-Transcriptional Regulation of the Cystic Fibrosis Transmembrane conductance Regulator (CFTR) by miR-101 and miR-494 Specific Binding. Schrijver I, editor. PLoS One 2011;6:e26601.

[47] Amato F, Seia M, Giordano S, et al. Gene Mutation in MicroRNA Target Sites of CFTR Gene: A Novel Pathogenetic Mechanism in Cystic Fibrosis? Vij N, editor. PLoS One 2013;8:e60448.

[48] Amato F, Tomaiuolo R, Nici F, et al. Exploitation of a very small peptide nucleic acid as a new inhibitor of miR-509-3p involved in the regulation of cystic fibrosis disease-gene expression. Biomed Res Int 2014;2014:610718.

[49] Ramachandran S, Karp PH, Jiang P, et al. A microRNA network regulates expression and biosynthesis of wild-type and DeltaF508 mutant cystic fibrosis transmembrane conductance regulator. Proc Natl Acad Sci U. S. A. 2012;109:13362-13367.

[50] Kumar $\mathrm{P}$, Bhattacharyya S, Peters $\mathrm{KW}$, et al. miR-16 rescues F508del-CFTR function in native cystic fibrosis epithelial cells. Gene Ther 2015;22:908-916.

[51] Hassan T, McKiernan PJ, McElvaney NG, et al. Therapeutic modulation of miRNA for the treatment of proinflammatory lung diseases. Expert Rev Anti Infect Ther 2012;10:359-368.

[52] Vestbo J, Hurd SS, Agustí AG, et al. Global Strategy for the Diagnosis, Management, and Prevention of Chronic Obstructive Pulmonary Disease. Am J Respir Crit Care Med 2013;187:347365.

[53] Lozano R, Naghavi M, Foreman K, et al. Global and regional mortality from 235 causes of death 
for 20 age groups in 1990 and 2010: a systematic analysis for the Global Burden of Disease Study 2010. Lancet 2012;380:2095-2128.

[54] López-Campos JL, Tan W, Soriano JB. Global burden of COPD. Respirology 2016;21:14-23.

[55] Mannino DM, Buist AS. Global burden of COPD: risk factors, prevalence, and future trends. Lancet 2007;370:765-773.

[56] COPD, Awareness Month, NHLBI, NIH [Internet]. [cited 2017 Aug 2]. Available from: https://www.nhlbi.nih.gov/health/educational/copd/event-listing/awareness-month/materialsresources.htm.

[57] COPD | European Lung Foundation - ELF [Internet]. [cited 2017 Aug 9]. Available from: http://www.europeanlung.org/en/lung-disease-and-information/lung-diseases/copd.

[58] WHO | Causes of COPD. WHO. 2013;

[59] Rab A, Rowe SM, Raju SV, et al. Cigarette smoke and CFTR: implications in the pathogenesis of COPD. Am. J. Physiol. Lung Cell Mol Physiol 2013;305:L530-41.

[60] Cantin AM. Cystic Fibrosis Transmembrane Conductance Regulator. Implications in Cystic Fibrosis and Chronic Obstructive Pulmonary Disease. Ann Am Thorac Soc. 2016;13:S150-5.

[61] Raju SV, Lin VY, Liu L, et al. The Cystic Fibrosis Transmembrane Conductance Regulator Potentiator Ivacaftor Augments Mucociliary Clearance Abrogating Cystic Fibrosis Transmembrane Conductance Regulator Inhibition by Cigarette Smoke. Am J Respir Cell Mol Biol 2017;56:99-108.

[62] Welsh MJ. Cigarette smoke inhibition of ion transport in canine tracheal epithelium. J Clin Invest 1983;71:1614-1623.

[63] **Cantin AM, Hanrahan JW, Bilodeau G, et al. Cystic Fibrosis Transmembrane Conductance Regulator Function Is Suppressed in Cigarette Smokers. Am J Respir Crit Care Med 2006;173:1139-1144.

** One of the first reports of the effects of cigarette smoke on CFTR function in vitro, and also decribing 
impaired nasal potential differences in smokers.

[64] ** Dransfield MT, Wilhelm AM, Flanagan B, et al. Acquired Cystic Fibrosis Transmembrane Conductance Regulator Dysfunction in the Lower Airways in COPD. Chest 2013;144:498-506.

**Key paper describing CFTR dysfunction in the COPD lung

[65] Kreindler JL, Jackson AD, Kemp PA, et al. Inhibition of chloride secretion in human bronchial epithelial cells by cigarette smoke extract. AJP Lung Cell Mol Physiol 2005;288:L894-L902.

[66] Clunes LA, Davies CM, Coakley RD, et al. Cigarette smoke exposure induces CFTR internalization and insolubility, leading to airway surface liquid dehydration. FASEB J 2012;26:533-545.

[67] Sloane PA, Shastry S, Wilhelm A, et al. A Pharmacologic Approach to Acquired Cystic Fibrosis Transmembrane Conductance Regulator Dysfunction in Smoking Related Lung Disease. Kreindler JL, editor. PLoS One 2012;7:e39809.

[68] Innes AL, Woodruff PG, Ferrando RE, et al. Epithelial Mucin Stores Are Increased in the Large Airways of Smokers With Airflow Obstruction. Chest 2006;130:1102-1108.

[69] Mall MA. Unplugging mucus in cystic fibrosis and chronic obstructive pulmonary disease. Ann Am Thorac Soc 2016;13:S177-S185.

[70] Rasmussen JE, Sheridan JT, Polk W, et al. Cigarette Smoke-induced Ca ${ }^{2+}$ Release Leads to Cystic Fibrosis Transmembrane Conductance Regulator (CFTR) Dysfunction. J Biol Chem 2014;289:7671-7681.

[71] Johannesson B, Hirtz S, Schatterny J, et al. CFTR Regulates Early Pathogenesis of Chronic Obstructive Lung Disease in $\beta E N a C$-Overexpressing Mice. Vij N, editor. PLoS One 2012;7:e44059.

[72] Ghosh A, Boucher RC, Tarran R. Airway hydration and COPD. Cell Mol Life Sci 2015;72:36373652.

[73] Button B, Anderson WH, Boucher RC. Mucus Hyperconcentration as a Unifying Aspect of the Chronic Bronchitic Phenotype. Ann Am Thorac Soc 2016;13 Suppl 2:S156-62. 
[74] Ballard ST, Trout L, Bebök Z, et al. CFTR involvement in chloride, bicarbonate, and liquid secretion by airway submucosal glands. Am J Physiol 1999;277:L694-9.

[75] Hug MJ, Tamada T, Bridges RJ. CFTR and Bicarbonate Secretion to Epithelial Cells. Physiology 2003;18:38-42.

[76] Bodas M, Min T, Vij N. Critical role of CFTR-dependent lipid rafts in cigarette smoke-induced lung epithelial injury. Am J Physiol Lung Cell Mol Physiol 2011;300:L811-20.

[77] Ni I, Ji C, Vij N. Second-Hand Cigarette Smoke Impairs Bacterial Phagocytosis in Macrophages by Modulating CFTR Dependent Lipid-Rafts. Chu HW, editor. PLoS One. 2015;10:e0121200.

[78] Raju SV, Jackson PL, Courville CA, et al. Cigarette Smoke Induces Systemic Defects in Cystic Fibrosis Transmembrane Conductance Regulator Function. Am J Respir Crit Care Med 2013;188:1321-1330.

[79] Rennolds J, Butler S, Maloney K, et al. Cadmium Regulates the Expression of the CFTR Chloride Channel in Human Airway Epithelial Cells. Toxicol Sci 2010;116:349-358.

[80] Hassan F, Nuovo GJ, Crawford M, et al. MiR-101 and miR-144 Regulate the Expression of the CFTR Chloride Channel in the Lung. Kreindler JL, editor. PLoS One. 2012;7:e50837.

[81] Hassan F, Xu X, Nuovo G, et al. Accumulation of metals in GOLD4 COPD lungs is associated with decreased CFTR levels. Respir Res 2014;15:69.

[82] Le Gars M, Descamps D, Roussel D, et al. Neutrophil Elastase Degrades Cystic Fibrosis Transmembrane Conductance Regulator via Calpains and Disables Channel Function In Vitro and In Vivo. Am J Respir Crit Care Med 2013;187:170-179.

[83] Alexander NS, Blount A, Zhang S, et al. Cystic fibrosis transmembrane conductance regulator modulation by the tobacco smoke toxin acrolein. Laryngoscope 2012;122:1193-1197.

[84] Moran AR, Norimatsu Y, Dawson DC, et al. Aqueous cigarette smoke extract induces a voltagedependent inhibition of CFTR expressed in Xenopus oocytes. AJP Lung Cell Mol Physiol 
2014;306:L284-L291.

[85] Gao L, Kim KJ, Yankaskas JR, et al. Abnormal glutathione transport in cystic fibrosis airway epithelia. Am J Physiol 1999;277:L113-8.

[86] Church DF, Pryor WA. Free-radical chemistry of cigarette smoke and its toxicological implications. Environ. Health Perspect 1985;64:111-126.

[87] René C, Lopez E, Claustres M, et al. NF-E2-related factor 2, a key inducer of antioxidant defenses, negatively regulates the CFTR transcription. Cell Mol Life Sci 2010;67:2297-2309.

[88] Zhang Z, Leir S-H, Harris A. Oxidative Stress Regulates CFTR Gene Expression in Human Airway Epithelial Cells through a Distal Antioxidant Response Element. Am J Respir Cell Mol Biol 2015;52:387-396.

[89] McKiernan PJ, Greene CM. MicroRNA Dysregulation in Cystic Fibrosis. Mediators Inflamm 2015;2015:529642.

[90] Ezzie ME, Crawford M, Cho J-H, et al. Gene expression networks in COPD: microRNA and mRNA regulation. Thorax 2012;67:122-131.

[91] Savitski AN, Mesaros C, Blair IA, et al. Secondhand smoke inhibits both $\mathrm{Cl}$ - and $\mathrm{K}+$ conductances in normal human bronchial epithelial cells. Respir Res 2009;10:120.

[92] Lambert JA, Raju SV, Tang LP, et al. Cystic Fibrosis Transmembrane Conductance Regulator Activation by Roflumilast Contributes to Therapeutic Benefit in Chronic Bronchitis. Am J Respir Cell Mol Biol 2014;50:549-558.

[93] Tyrrell J, Qian X, Freire J, et al. Roflumilast combined with adenosine increases mucosal hydration in human airway epithelial cultures after cigarette smoke exposure. Am J Physiol Lung Cell Mol Physiol 2015;308:L1068-77.

[94] Schmid A, Baumlin N, Ivonnet P, et al. Roflumilast partially reverses smoke-induced mucociliary dysfunction. Respir Res 2015;16:135. 
[95] Dekkers JF, van der Ent CK, Beekman JM. Novel opportunities for CFTR-targeting drug development using organoids. Rare Dis 2013;1:e27112.

[96] Dekkers JF, Wiegerinck CL, de Jonge HR, et al. A functional CFTR assay using primary cystic fibrosis intestinal organoids. Nat Med 2013;19:939-945.

[97] Okiyoneda T, Veit G, Dekkers JF, et al. Mechanism-based corrector combination restores $\Delta \mathrm{F} 508$ CFTR folding and function. Nat Chem Biol 2013;9:444-454.

[98] Schwank G, Koo B-K, Sasselli V, et al. Functional repair of CFTR by CRISPR/Cas9 in intestinal stem cell organoids of cystic fibrosis patients. Cell Stem Cell 2013;13:653-658.

[99] Beekman JM. Individualized medicine using intestinal responses to CFTR potentiators and correctors. Pediatr Pulmonol 2016;51:S23-S34.

[100] Dekkers JF, Gogorza Gondra RA, Kruisselbrink E, et al. Optimal correction of distinct CFTR folding mutants in rectal cystic fibrosis organoids. Eur Respir J 2016;48:451-458.

[101] Dekkers R, Vijftigschild LAW, Vonk AM, et al. A bioassay using intestinal organoids to measure CFTR modulators in human plasma. J Cyst Fibros 2015;14:178-181.

[102] Noordhoek J, Gulmans V, van der Ent K, et al. Intestinal organoids and personalized medicine in cystic fibrosis. Curr Opin Pulm Med 2016;22:610-616.

[103] Zomer-van Ommen DD, Vijftigschild LAW, Kruisselbrink E, et al. Limited premature termination codon suppression by read-through agents in cystic fibrosis intestinal organoids. J Cyst Fibros 2016;15:158-162.

[104] *Guimbellot JS, Leach JM, Chaudhry IG, et al. Nasospheroids permit measurements of CFTRdependent fluid transport. JCI Insight. 2017;2.

**Newest ex vivo model for testing CFTR function and drug responses.

[105] Aasen T, Raya A, Barrero MJ, et al. Efficient and rapid generation of induced pluripotent stem cells from human keratinocytes. Nat. Biotechnol 2008;26:1276-1284. 
[106] Haase A, Olmer R, Schwanke K, et al. Generation of Induced Pluripotent Stem Cells from Human Cord Blood. Cell Stem Cell 2009;5:434-441.

[107] Lachmann N, Happle C, Ackermann M, et al. Gene Correction of Human Induced Pluripotent Stem Cells Repairs the Cellular Phenotype in Pulmonary Alveolar Proteinosis. Am J Respir Crit Care Med 2013;189:131126070906004.

[108] Katsirntaki K, Mauritz C, Olmer R, et al. Bronchoalveolar sublineage specification of pluripotent stem cells: effect of dexamethasone plus cAMP-elevating agents and keratinocyte growth factor. Tissue Eng Part A. 2015;21:669-682.

[109] Huang SXL, Islam MN, O'Neill J, et al. Efficient generation of lung and airway epithelial cells from human pluripotent stem cells. Nat Biotechnol 2014;32:84-91.

[110] Schmeckebier S, Mauritz C, Katsirntaki K, et al. Keratinocyte growth factor and dexamethasone plus elevated cAMP levels synergistically support pluripotent stem cell differentiation into alveolar epithelial type II cells. Tissue Eng Part A. 2013;19:938-951. 
Table 1. Major differences and similarities between cystic fibrosis and COPD

\begin{tabular}{|c|c|c|}
\hline FEATURES & CYSTIC FIBROSIS & COPD \\
\hline \multicolumn{3}{|l|}{ MAJOR DIFFERENCES } \\
\hline Etiology & Inherited disease & $\begin{array}{l}\text { Acquired multifactorial } \\
\text { disease }\end{array}$ \\
\hline CFTR dysfunction & CFTR gene mutations & $\begin{array}{l}\text { Acquired CFTR dysfunction } \\
\text { (mainly smoking-induced) }\end{array}$ \\
\hline Lung disease onset & $\begin{array}{l}\text { Usually starts early in } \\
\text { childhood }\end{array}$ & $\begin{array}{l}\text { Clinical manifestations start } \\
>40 \text { years of age. } \\
\text { Prevalence increases with } \\
\text { age }\end{array}$ \\
\hline $\begin{array}{l}\text { Clinical phenotypic } \\
\text { features }\end{array}$ & $\begin{array}{l}\text { Bronchiectasis } \\
\text { Chronic airway infection }\end{array}$ & $\begin{array}{l}\text { Chronic bronchitis and } \\
\text { emphysema; } \\
\text { bronchiectasis less common } \\
\text { Chronic airway infection less } \\
\text { common }\end{array}$ \\
\hline \multicolumn{3}{|l|}{ MAJOR SIMILARITIES } \\
\hline Clinical features & \multicolumn{2}{|c|}{$\begin{array}{l}\text { Chronic progressive lung disease } \\
\text { Irreversible airflow obstruction } \\
\text { Recurrent infectious exacerbations }\end{array}$} \\
\hline Pathologic features & \multicolumn{2}{|c|}{$\begin{array}{c}\text { Mucus hypersecretion and plugging } \\
\text { ASL dehydration } \\
\text { Chronic airway inflammation } \\
\text { Tissue damage and remodelling }\end{array}$} \\
\hline
\end{tabular}


